\title{
Event-Triggered Strategies for Decentralized Model Predictive Controllers
}

\author{
Alina Eqtami* Dimos V. Dimarogonas ** \\ Kostas J. Kyriakopoulos*
}

\author{
* Control Systems Lab, Department of Mechanical Engineering, \\ National Technical University of Athens, 9 Heroon Polytechniou \\ Street, Zografou 15780, Greece (e-mail:alina,kkyria@mail.ntua.gr). \\ ** School of Electrical Engineering, Royal Institute of Technology \\ (KTH),SE-100 44, Stockholm, Sweden(e-mail:dimos@ee.kth.se).
}

\begin{abstract}
In this paper, novel event-triggered strategies for the design of model predictive (MPC) controllers are presented. The MPC framework consists in finding the solution to a constraint optimal-control problem at every time-step. The case of triggering the optimization of the MPC only when is needed, is investigated. The centralized case is treated first and the results are then extended to a decentralized formulation. We consider a system composed by a number of interconnected subsystems, each one of them controlled by a robust MPC algorithm. Using the Input-to-State (ISS) property of the decentralized MPC controller we reach to an event-triggering rule, for each of the subsystems.
\end{abstract}

\section{INTRODUCTION}

Decentralized control is an efficient formulation for controlling large scale systems, while model predictive control formulations have the capability to deal with nonlinearities and state and control constraints. Therefore, decentralized approaches to MPC have gained much interest in recent years. Related results on decentralized MPC can be found in Dunbar et al. [2006], Franco et al. [2008], Keviczky et al. [2006], Raimondo et al. [2007], Raimondo et al. [2009] and in the review paper Scattolini [2009] and the number of papers quoted therein.

One of the aspects that should be taken in consideration when implementing decentralized control laws, is the communication and controller schemes. The scheduling of the actuation updates can be done in a time-driven or in an event-driven fashion. The first one might be proven a conservative choice, while when we have limited resources, the second choice may be more favorable. In recent years the framework of event-driven feedback and sampling has been developed. Related works can be found in Heemels et al. [2007] and Tabuada [2007] for general nonlinear systems, while similar approaches for decentralized frameworks can be found in Dimarogonas et al. [2009], Mazo et al. [2010], and Wang et al. [2009].

The main assumption used for these event-triggered policies, is the Input-to-State property of the plant. There has been a lot of research on ISS properties of the MPC framework for general discrete-time systems. Recent results can be found in Lazar et al. [2009] and Pin et al. [2008]. In this work, in order to find a triggering condition for sampling in the centralized case, we appropriately modify the formulation presented in Marruedo et al. [2002]. The ISS property of the system under decentralized MPC controllers is discussed in Franco et al. [2008], Raimondo et al. [2009] and Raimondo et al. [2007]. Here we employ the ISS property of the decentralized MPC in Raimondo et al. [2007] in order to reach to a triggering condition for each of the subsystems of the framework.

The contribution of this paper relies in finding a triggering rule for sampling in the case of nonlinear systems, under a nonlinear MPC strategy. In the aforementioned eventtriggering techniques, the control value is held constant between the actuator updates. Nevertheless, in this paper the fact that predictive controllers provide a control sequence at each time-step is used. In particular, the control sequence that is provided by the MPC controller is applied in an open-loop fashion between the actuator updates. First, this event-triggering scheme is applied to a centralized MPC controller. Then, we consider a system composed by a number of interconnected subsystems, each one of them controlled by a robust MPC algorithm. Using this decentralized MPC controller we derive an eventtriggering rule for each of the subsystems.

Although the event-triggering setup for MPC controllers is quite new, some results have already been presented, Varutti et al. [2009], Iino et al. [2009], Grune et al. [2009] and Sijs et al. [2010]. Related to the above references, we provide here a different event triggering strategy to compute the event times.

The remainder of the paper is organized as follows. In Section 2 and in Section 3, the problem statement and the triggering condition for the centralized case is presented respectively. The decentralized counterpart is presented in the following two Sections; the problem statement for the decentralized framework is provided in Section 4, while in Section 5 the triggering rule is given. Section 6 summarizes the results of this paper and indicates further research efforts. 


\section{PROBLEM STATEMENT FOR THE CENTRALIZED NMPC CASE}

In order to find a triggering condition for the discretetime nonlinear system under an NMPC control law, a modification of the analysis proposed in Marruedo et al. [2002], will be used in the following, in order to find a triggering condition.

Consider the nonlinear discrete-time dynamic system

$$
x_{k+1}=f\left(x_{k}, u_{k}\right)+w_{k}
$$

where $x_{k} \in \mathbb{R}^{n}$ denotes the system's state, $u_{k} \in \mathbb{R}^{m}$ the control variables and $w_{k} \in \mathcal{W} \subseteq \mathbb{R}^{n}$ is the additive disturbance. Assume that $\mathcal{W}$ is a compact set, containing the origin, and that the admissible set of uncertainties are bounded, thus

$$
w_{k} \in \mathcal{W}, \quad\left\|w_{k}\right\| \leq \gamma
$$

The state and control variables are subjected to the following constraints

$$
x_{k} \in \mathcal{X}, \quad u_{k} \in \mathcal{U}, \quad k \in \mathbb{Z}_{\geq 0}
$$

For control design purposes, we assume the nominal model of the system is of the form

$$
x_{k+1}=f\left(x_{k}, u_{k}\right)
$$

Assume that $f(0,0)=0$ and that $f(x, u)$ is locally Lipschitz in $x$ in the domain $\mathcal{X} \times \mathcal{U}$, with Lipschitz constant $L_{f}$. Given the system (4), the following double subscript notation will be used hereafter

$$
\hat{x}(k+j+1 \mid k)=f\left(\hat{x}(k+j \mid k), u_{k+j}\right)
$$

where $\hat{x}(\cdot)$ is the predicted state and $\hat{x}(k+j+1 \mid k)$ is the predicted state at time $k+j+1$, based on the measured state of the real system at time $k, u_{k+j}$ is a control sequence for time $k$ until $k+j$, and $x_{k}=\hat{x}(k \mid k)$ is the measured state at time step $k$.

The uncertainty of the system can cause discrepancies between the actual state trajectory of the system, given from (1), subject to a specific sequence of inputs and the predicted state given from (4), for the same sequence of inputs. In order to account for this mismatch the error $e$, is introduced in the analysis. The error $e$ is defined as the norm of the difference between the predicted and the real evolution of the state. In the sequel, the double subscript notation will be reserved for the error. The error $e(k+j \mid k)$ will thus be given by

$$
e(k+j \mid k)=\left\|x_{k+j}-\hat{x}(k+j \mid k)\right\|
$$

Note, that the sequence of states of the actual system is thought to be measurable. It can be proven that the error $e(k+j \mid k)$ is bounded, and that it depends on the error at time $k+1$, i.e. for a given sequence of inputs the error $e(k+j \mid k)$, at time step $k+j$, will satisfy

$$
e(k+j \mid k) \leq L_{f}^{j-1} \cdot e(k+1 \mid k)+\frac{L_{f}^{j-1}-1}{L_{f}-1} \cdot \gamma
$$

\subsection{NMPC for Discrete-Time Systems}

NMPC involves solving on-line a finite-horizon, openloop optimal control problem (abbr. OCP), based on the measurement provided by the plant. The OCP in the discrete-time case, consists in minimizing, with respect to a control sequence $u_{F}(k) \triangleq[u(k \mid k), u(k+1 \mid k), \ldots, u(k+N-$
$1 \mid k)$ ], a cost function $J_{N}\left(x_{k}, u_{F}(k)\right)$. The positive integer $N \in \mathbb{Z}_{\geq 0}$, denotes the prediction horizon. Thus, the OCP for the nominal system (4), is given by

$$
\begin{aligned}
& \min _{u_{F}(k)} J_{N}\left(x_{k}, u_{F}(k)\right)= \\
& \min _{u_{F}(k)} \sum_{i=0}^{i=N-1} L(\tilde{x}(k+i \mid k), u(k+i \mid k)) \\
& +V(\tilde{x}(k+N \mid k))
\end{aligned}
$$

s.t.

$$
\begin{aligned}
& \tilde{x}(k+j \mid k) \in \mathcal{X}_{j} \quad \forall j=1, \ldots, N-1 \\
& u(k+j \mid k) \in \mathcal{U} \quad \forall j=0, \ldots, N-1 \\
& \tilde{x}(k+N \mid k) \in \mathcal{X}_{f}
\end{aligned}
$$

where $\mathcal{X}_{f}$ denotes the terminal constraint set and $\sim$ denotes the controller internal variables and $\tilde{x}(k \mid k)=x_{k}$.

Following a similar procedure as in Marruedo et al. [2002], the following assumptions for the stage cost $L(\cdot)$ and the terminal cost $V(\cdot)$ from $(7 \mathrm{a})$, are stated

Assumption 1. i) The stage cost $L(x, u)$ is Lipschitz continuous in $\mathcal{X} \times \mathcal{U}$, with a Lipschitz constant $L_{c}$. Assume that $L(0,0)=0$ and that there are positive integers $\alpha>0$ and $\omega \geq 1$, such that $L(x, u) \geq \alpha\|(x, u)\|^{\omega}$.

ii) Let the terminal region $\mathcal{X}_{f}$ from $(7 \mathrm{~d})$ be a subset of an admissible positively invariant set $\Phi$ of the nominal system. Assume that there is a local stabilizing controller $h\left(x_{k}\right)$ for the terminal state $\mathcal{X}_{f}$. The associated Lyapunov function $V(\cdot)$ has the following properties $V\left(f\left(x_{k}, h\left(x_{k}\right)\right)\right.$ $V\left(x_{k}\right) \leq-L\left(x_{k}, h\left(x_{k}\right)\right), \forall x_{k} \in \Phi$, and is Lipschitz in $\Phi$, with Lipschitz constant $L_{V}$. The set $\Phi$ is given by $\Phi=\left\{x \in \mathbb{R}^{n}: V(x) \leq \alpha_{\Phi}\right\}$ such that $\Phi \subseteq \mathcal{X}^{h}=\{x \in$ $\left.\mathcal{X}_{N-1}: h(x) \in \mathcal{U}\right\}$. The set $\mathcal{X}_{f}=\left\{x \in \mathbb{R}^{n}: \bar{V}(x) \leq \alpha_{\nu}\right\}$ is such that for all $x \in \Phi, f(x, h(x)) \in \mathcal{X}_{f}$.

The state constraint set $\mathcal{X}$ of the standard MPC formulation, is being replaced by a restricted constraint set $\mathcal{X}_{j}$ from (7b). This state constraints' tightening for the nominal system with additive disturbance, guarantees that the evolution of the real system will be admissible for all time. Using the supremum of (6), there is $\mathcal{X}_{j}=\mathcal{X} \sim \mathscr{B}_{j}$ where $\mathscr{B}_{j}=\left\{x \in \mathbb{R}^{n}:\|x\| \leq \frac{L_{f}^{j-1}-1}{L_{f}-1} \cdot \gamma\right\}$. The set operator " $\sim$ " denotes the Pontryagin difference.

\section{TRIGGERING CONDITION FOR THE CENTRALIZED NMPC CASE}

In this section the triggering condition for the discretetime system under the NMPC control will be provided. Before tackling this problem, some general concepts about using the ISS property of a system in order to find a triggering condition, are going to be presented first.

A continuous function $V: \mathbb{R}^{n} \rightarrow \mathbb{R}_{\geqslant} 0$ is an ISS-Lyapunov function for a system of the form $x_{k+1}=f\left(x_{k}, e_{k}\right)$, if there exist $\mathcal{K}_{\infty}$ functions $\alpha_{1}, \alpha_{2}$, such that $\alpha_{1}\left(\left\|x_{k}\right\|\right) \leq V\left(x_{k}\right) \leq$ $\alpha_{2}\left(\left\|x_{k}\right\|\right)$ for all $x \in \mathcal{X}$ and for some class $\mathcal{K}_{\infty}$ function, $\alpha_{3}$, and some class $\mathcal{K}, \alpha_{4}$, function $V\left(x_{k}\right)$ also satisfies

$$
V\left(f\left(x_{k}, e_{k}\right)\right)-V\left(x_{k}\right) \leq-\alpha_{3}\left(\left\|x_{k}\right\|\right)+\alpha_{4}\left(\left\|e_{k}\right\|\right)
$$

In this case the system is ISS with respect to the measurement error $e_{k}$. System remains stable, if $e_{k}$ satisfies

$$
\alpha_{4}\left(\left\|e_{k}\right\|\right) \leq \sigma \alpha_{3}\left(\left\|x_{k}\right\|\right)
$$


with $0<\sigma<1$, because invoking this rule into equation (8), $V$ is still guaranteed to be decreasing. A triggering condition is thus given by (9) as stated in Eqtami et al. [2010]. Once the error is large enough so that (9) is violated, the control law must be updated and the error is reset to zero. Hence, $V$ remains strictly decreasing under the triggering rule.

\subsection{Feasibility and ISS Stability}

In the classic discrete-time NMPC strategy the control law is updated at each time-step $k$. The control law is given by $u_{k}=u^{*}(k \mid k)$, where $u^{*}(k \mid k)$ is the first term of the optimal solution provided by the discrete-time OCP (7a)(7d). In the event-triggered setup the rest of the optimal sequence might be used provided that some conditions are fulfilled. In this case the optimal control sequence is recalculated at the discrete time instants $\left\{t_{0}, t_{1}, t_{2}, \ldots\right\} \subseteq$ $\left\{k_{0}, k_{1}, k_{2}, \ldots\right\}$. Assume that for every triggering instant $t_{i}$ a new OCP is triggered too, and that $t_{i}$ coincides with $k_{j}$. During the time span $\left[t_{i}, t_{i+1}\right)$, where $t_{i+1}$ is the next triggering instant when the new OCP is triggered, the control law provided at $t_{i} \equiv k_{j}$ is implemented in openloop fashion. The triggering condition is going to be stated later in the text.

In the NMPC case considered here, consider control trajectories $\bar{u}_{F}(k+m)$, for time steps $m=0, \ldots, N-1$, based on the optimal solution in $k-1, u_{F}^{*}(k-1)$, i.e. for $m=0, \ldots, N-1$

$$
\begin{aligned}
& \bar{u}(k+j \mid k+m)= \\
& =\left\{\begin{array}{cl}
u^{*}(k+j \mid k-1) & \text { for } j=m, \ldots, N-2 \\
h(\hat{x}(k+N-1 \mid k+m)) & \text { for } j=m+N-1
\end{array}\right.
\end{aligned}
$$

From feasibility of $u_{F}^{*}(k-1)$ it follows that for $m=$ $0, \ldots, N-1$ there is $\bar{u}(k+j \mid k+m) \in \mathcal{U}$, and according to Marruedo et al. [2002] $\hat{x}(k+N \mid k+m) \in \mathcal{X}_{f}$, for all $m=0, \ldots, N-1$, provided that the uncertainties are bounded by $\gamma \leq\left(\alpha_{\Phi}-\alpha_{v}\right) /\left(L_{V} \cdot L_{f}^{N-1}\right)$.

The optimal cost at time step $k-1$ is $J_{N}^{*}(k-1)$ and the cost of the feasible sequence at a time step $j \in[0, N-1]$ is indicated by $\bar{J}_{N}(k+j)$. Then the difference of these costs is

$$
\Delta J_{j}=\bar{J}_{N}(k+j)-J_{N}^{*}(k-1)
$$

The next theorem can now be stated

Theorem 1. Consider the system (1) subject to (3) and assume that the previously presented Assumption 1 holds. Then, using the control law from (10), the difference between the cost of a feasible sequence at time step $k+j$ and the optimal cost of at time step $k-1$ is bounded by

$$
\Delta J_{j} \leq L_{Z_{j}} \cdot e(k+j \mid k-1)-\alpha \sum_{i=0}^{j}\left\|x_{k-i+j}\right\|^{\omega}
$$

where $L_{Z_{j}}$ is given by

$$
L_{Z_{j}}=L_{V} L_{f}^{(N-1)-j}+L_{C} \cdot \frac{L_{f}^{(N-1)-j}-1}{L_{f}-1}
$$

Proof. First, the difference (11) is calculated for $j=0$. A similar procedure as in Marruedo et al. [2002] is going to be used. Then the calculation will be repeated for $j=1$, and finally the general rule for random $j$ will be stated. For $j=0$ the difference (11) is

$$
\begin{aligned}
\Delta J_{0} & =\bar{J}_{N}(k)-J_{N}^{*}(k-1) \\
& =\sum_{i=0}^{N-1}\{L(\bar{x}(k+i \mid k), \bar{u}(k+i \mid k)) \\
& \left.-L\left(\hat{x}(k+i-1 \mid k-1), u^{*}(k+i-1 \mid k-1)\right)\right\} \\
& +V(\bar{x}(k+N \mid k))-V(\hat{x}(k+N-1 \mid k-1)) \\
& =\sum_{i=0}^{N-2}\{L(\bar{x}(k+i \mid k), \bar{u}(k+i \mid k)) \\
& \left.-L\left(\hat{x}(k+i \mid k-1), u^{*}(k+i \mid k-1)\right)\right\} \\
& +L(\bar{x}(k+N-1 \mid k), h(\bar{x}(k+N-1 \mid k)) \\
& -L\left(x_{k-1}, u_{k-1}\right) \\
& +V(\bar{x}(k+N \mid k))-V(\hat{x}(k+N-1 \mid k-1))
\end{aligned}
$$

From definition of (10) we have $\bar{u}(k+i \mid k)=u^{*}(k+i \mid k-1)$. Imposing the control law (10) for $m=0$ to the system, by induction we get

$$
\|\hat{x}(k+j \mid k)-\hat{x}(k+j \mid k-1)\| \leq L_{f}^{j} \cdot e(k \mid k-1)
$$

where the error $e(k \mid k-1)$, using (5), is given by

$$
e(k \mid k-1)=\left\|x_{k}-\hat{x}(k \mid k-1)\right\|
$$

The difference between the running costs, with the help of (14) and (15), is

$$
\begin{aligned}
& \quad L(\bar{x}(k+j \mid k), \bar{u}(k+j \mid k)) \\
& -L\left(\hat{x}(k+j \mid k-1), u^{*}(k+j \mid k-1)\right) \leq L_{c} \cdot L_{f}^{j} \cdot e(k \mid k-1) \\
& \text { Analogously, } \\
& \quad V(\bar{x}(k+N-1 \mid k))-V(\hat{x}(k+N-1 \mid k-1)) \\
& \quad \leq L_{V} \cdot L_{f}^{N-1} \cdot e(k \mid k-1)
\end{aligned}
$$

Consider also, that from Assumption 1i) we have $L(x, u) \geq$ $\alpha\|(x, u)\|^{\omega} \geq \alpha\|x\|^{\omega}$. Substituting these expressions to (13), the following is derived

$$
\Delta J_{0} \leq L_{Z_{0}} \cdot e(k \mid k-1)-\alpha\left\|x_{k-1}\right\|^{\omega}
$$

with $L_{Z_{0}}=L_{V} \cdot L_{f}^{N-1}+L_{c} \cdot \frac{L_{f}^{N-1}-1}{L_{f}-1}$.

For $j=1$ the difference (11) becomes

$$
\begin{aligned}
\Delta J_{1} & =\bar{J}_{N}(k+1)-J_{N}^{*}(k-1) \\
& =\sum_{i=0}^{N-1}\{L(\bar{x}(k+i+1 \mid k+1), \bar{u}(k+i+1 \mid k+1)) \\
& \left.-L\left(\hat{x}(k+i-1 \mid k-1), u^{*}(k+i-1 \mid k-1)\right)\right\} \\
& +V(\bar{x}(k+N+1 \mid k+1))-V(\hat{x}(k+N-1 \mid k-1)) \\
& =\sum_{i=0}^{N-3}\{L(\bar{x}(k+i+1 \mid k+1), \bar{u}(k+i+1 \mid k+1)) \\
& \left.-L\left(\hat{x}(k+i+1 \mid k-1), u^{*}(k+i+1 \mid k-1)\right)\right\} \\
& +L(\bar{x}(k+N-1 \mid k+1), h(\bar{x}(k+N-1 \mid k+1)) \\
& -L\left(x_{k-1}, u_{k-1}\right) \\
& +L(\bar{x}(k+N \mid k+1), h(\bar{x}(k+N \mid k+1)) \\
& -L\left(x_{k}, u_{k}\right) \\
& +V(\bar{x}(k+N+1 \mid k+1)) \\
& -V(\hat{x}(k+N-1 \mid k-1)) \\
& +V(\bar{x}(k+N \mid k+1) \\
& -V(\bar{x}(k+N \mid k+1)
\end{aligned}
$$

From Assumption 1ii), we have 


$$
\begin{aligned}
& L(\bar{x}(k+N \mid k+1), h(\bar{x}(k+N \mid k+1))) \\
& +V(\bar{x}(k+N+1 \mid k+1))-V(\bar{x}(k+N \mid k+1)) \leq 0
\end{aligned}
$$

Moreover,

$$
\begin{aligned}
& V(\bar{x}(k+N-1 \mid k+1))-V(\hat{x}(k+N-1 \mid k-1)) \leq \\
& L_{V} \cdot L_{f}^{N-2} \cdot e(k+1 \mid k-1)
\end{aligned}
$$

Substituting these expressions to (19), it can be concluded that the difference $\Delta J_{1}$ is bounded by

$$
\Delta J_{1} \leq L_{Z_{1}} \cdot e(k+1 \mid k-1)-\alpha|| x_{k-1}\left\|^{\omega}-\alpha\right\| x_{k} \|^{\omega}
$$

with $L_{Z_{1}}=L_{V} \cdot L_{f}^{N-2}+L_{c} \cdot \frac{L_{f}^{N-2}-1}{L_{f}-1}$. From the above it can be concluded using the same calculation, that for random $j \in[0, N-1]$ the difference $\Delta J_{j}=\bar{J}_{N}(k+j)-J_{N}^{*}(k-1)$, is given from (12), and hence the proof is completed.

\subsection{Triggering Condition}

System (1), subject to (3), which satisfies the Assumption 1, is ISS stable with respect to measurement errors, under an NMPC strategy. This can be concluded by the optimality of the solution that results to

$J_{N}^{*}(k)-J_{N}^{*}(k-1) \leq \Delta J_{0} \leq L_{Z_{0}} \cdot e(k \mid k-1)-\alpha|| x_{k-1} \|^{\omega}$

Using this ISS property of the system, and based on the general rule given by (9), the triggering rule for discretetime systems (1) is written in this case as

$$
L_{Z_{0}} \cdot e(k \mid k-1) \leq \sigma \cdot \alpha\left\|x_{k-1}\right\|^{\omega}
$$

The next OCP is thus triggered if condition (23) is violated, otherwise the control law from (10) is used for $m=0$.

The triggering rule (23), is valid in the first step. In order to ensure that the system stays stable, using control law (10), for $m \geq 0$, there are few more things to consider. According to Marruedo et al. [2002] and the proof of Theorem 1, optimality of the solution is not necessary to guarantee convergence of the closed-loop system. Thus, in order to maintain stability we must ensure that $\Delta J_{j}$ is strictly decreasing for all $m \geq 0$. The system can use the control law (10), as long as

$$
\Delta J_{j+1} \leq \Delta J_{j}
$$

In this case the convergence of the closed-loop system is guaranteed.

Consequently, the triggering rule can be stated as

$$
L_{Z_{j}} \cdot e(k+j \mid k-1) \leq \sigma \cdot \alpha \cdot \sum_{i=0}^{j}\left\|x_{k-i+j}\right\|^{\omega}
$$

and

$$
\begin{array}{r}
L_{Z_{j}} \cdot e(k+j \mid k-1)-\sigma \cdot \alpha \cdot \sum_{i=0}^{j}\left\|x_{k-i+j}\right\|^{\omega} \leq \\
L_{Z_{j-1}} \cdot e(k+j-1 \mid k-1)-\sigma \cdot \alpha \cdot \sum_{i=0}^{j-1}\left\|x_{k-i+j}\right\|^{\omega}
\end{array}
$$

The next OCP is triggered whenever condition (25a) or (25b) is violated. Note, that the state vector $x_{k}$ is assumed to be available through measurements and that it provides the current plant information.

The previous analysis guarantees that the closed loop system will have the same convergence properties as in
Marruedo et al. [2002]. However, the OCP in the case of this paper is not calculated at each time instant, but only when the triggering condition is violated. Thus the convergence to a compact set and ultimate boundedness properties of Marruedo et al. [2002] are preserved in the event-triggered formulation:

Theorem 2. Consider the system (1), subject to (3) under an NMPC strategy and assume that the previously presented Assumption 1 holds. Then the NMPC control law given by (7a)-(7d) along with the triggering rule (25a)(25b), drives the closed loop system towards a compact set where it is ultimately bounded.

\section{PROBLEM STATEMENT FOR THE DECENTRALIZED NMPC CASE}

In the following, the proposed framework for finding eventtriggering condition for sampling is extended to a general discrete-time system which is composed by the interconnection of $M$ local subsystems, controlled by a decentralized NMPC control law. The framework is considered to be fully decentralized, i.e., there is no information exchange between the subsystems.

A stabilizing decentralized MPC algorithm of nonlinear systems composed by a number of subsystems has been presented in Raimondo et al. [2007]. Each subsystem is locally controlled by an NMPC controller with guaranteed ISS property. Considering the effect of interconnections as perturbation terms it is shown that the overall system is also ISS. In accordance with the previous centralized approach, a modification of the analysis in Raimondo et al. [2007] is going to be equipped in the sequel, in order to derive the event-triggering condition of the system.

Consider the nonlinear perturbed discrete-time dynamic subsystem

$$
x_{s}(k+1)=f_{s}\left(x_{s}(k), u_{s}(k)\right)+g_{s}(x(k))+\psi_{s}(k)
$$

with $k \in \mathbb{Z}_{>0}$ and $s=1, \ldots, M$. The state of the $s$ th subsystem is $x_{s}(k) \in \mathbb{R}^{n_{s}}$ while $u_{s}(k) \in \mathbb{R}^{m_{s}}$ is the control variable and finally $\psi_{s}(k) \in \mathbb{R}^{n_{s}}$ is the additive uncertainty. Assume also that $f_{s}(0,0)=0$. The overall state is given as

$$
x(k) \triangleq\left[x_{1}(k), x_{2}(k), \ldots, x_{M}(k)\right] \in \mathbb{R}^{n}
$$

with $n=\sum_{s=1}^{M} n_{s}$. Notice that $g_{s}$ depends on the overall state and describes the influence of $M$ subsystems on the $s$-subsystem and is such that $g_{s}(0)=0$. The state and the disturbance are required to fulfil the following constraints

$$
x_{s} \in \mathcal{X}_{s} \quad u_{s}(k) \in \mathcal{U}_{s} \quad \psi_{s} \in \Psi_{s}
$$

where $\mathcal{X}_{s}$ and $\Psi_{s}$ are compact sets of $\mathbb{R}^{n_{s}}$ and $\mathcal{U}_{s}$ is a compact set of $\mathbb{R}^{m_{s}}$ all of them containing the origin as an interior point. Defining $f(x, u) \triangleq\left[f_{1}\left(x_{1}, u_{1}\right), \ldots, f_{M}\left(x_{M}, u_{M}\right)\right]$, $g(x) \triangleq\left[g_{1}(x), \ldots, g_{M}(x)\right]$ and $\psi \triangleq\left[\psi_{1}, \ldots, \psi_{M}\right]$, the whole system can be written as

$$
x(k+1)=f(x(k), u(k))+g(x(k))+\psi(k)
$$

with $k \in \mathbb{Z}_{\geq 0}$.

Assumption 2: i) There exists positive Lipschitz constant $L_{f_{s}}$ such that $\left\|f_{s}\left(x_{1}, u_{s}\right)-f_{s}\left(x_{2}, u_{s}\right)\right\| \leqslant L_{f_{s}}\left\|x_{1}-x_{2}\right\|$, for all $x_{1}, x_{2} \in \mathcal{X}_{s}$ and for all $u_{s} \in \mathcal{U}_{s}$ and all $s=1, \ldots, M$.

ii) There exist $M$ positive constants $L_{g_{s}, j}$ with $s=$ $1, \ldots, M$, such that $\left\|g_{s}(x)\right\| \leq \sum_{j=1}^{M} L_{g_{s}, j}\left\|x_{j}\right\|$. 
iii) For each subsystem, the sum of interaction term and the disturbance term is restricted to fulfil the following constraint $w_{s} \triangleq\left\{g_{s}(x)+\psi_{s}\right\} \in \mathcal{W}_{s}$, for all $x \in \mathcal{X}$ and for all $\psi_{s} \in \Psi_{s}$, where $\mathcal{W}_{s}$ is a compact set of $\mathbb{R}^{n_{s}}$, containing the origin as an interior point, while $\mathcal{X} \triangleq \mathcal{X}_{1} \times \cdots \times \mathcal{X}_{M}$.

\subsection{NMPC for Decentralized Discrete-Time Systems}

The Optimal Control Problem (abbr. OCP) for each subsystem is solved for the nominal state dynamics of the system (26), i.e.,

$$
x_{s}(k+1)=f_{s}\left(x_{s}(k), u_{s}(k)\right)
$$

As in the centralized case, the OCP for the nominal system (30), is obtained by locally minimizing at time instant $k$ with respect to a control sequence $u_{s F}(k) \triangleq$ $\left[u_{s}(k \mid k), \ldots, u_{s}\left(k+N_{s}-1 \mid k\right)\right]$, the following performance index

$$
\begin{aligned}
& \min _{u_{s F}(k)} J_{s}\left(x_{s}, u_{s F}(k)\right)= \\
& \min _{u_{s F}(k)} \sum_{i=0}^{N_{s}-1} L_{s}\left(\tilde{x}_{s}(k+i \mid k), u_{s}(k+i \mid k)\right)+V_{s}\left(\tilde{x}_{s}\left(k+N_{s} \mid k\right)\right)
\end{aligned}
$$

s.t.

$$
\begin{aligned}
& \tilde{x}_{s}(k+j \mid k) \in \mathcal{X}_{j_{s}} \quad \forall j=1, \ldots, N_{s}-1 \\
& u_{s}(k+j \mid k) \in \mathcal{U}_{s} \quad \forall j=0, \ldots, N_{s}-1 \\
& \tilde{x}_{s}\left(k+N_{s} \mid k\right) \in \mathcal{X}_{f_{s}}
\end{aligned}
$$

where $\mathcal{X}_{f_{s}}$ denotes the terminal constraint set. As in the centralized case, the state constraint set is being replaced with a restricted set $\mathcal{X}_{j_{s}}$. Following a similar procedure as in Raimondo et al. [2007], we make the following assumptions for the stage cost $L_{s}(\cdot)$ and the terminal cost $V_{s}(\cdot)$ from $(31 \mathrm{a})$ :

Assumption 3: i) The stage cost $L_{s}\left(x_{s}, u_{s}\right)$ is Lipschitz continuous in $\mathcal{X}_{s} \times \mathcal{U}_{s}$, with a Lipschitz constant $L_{c_{s}}$. Let $L_{s}(0,0)=0$, and assume that $L_{s}\left(x_{s}, u_{s}\right) \geqslant \alpha_{s}\left(\left\|x_{s}\right\|\right)$ where $\alpha_{s}$ is a class $\mathcal{K}_{\infty}$-function.

ii) Let the terminal region $\mathcal{X}_{f_{s}}$ from $(31 \mathrm{~d})$ be a subset of an admissible positively invariant set $\mathcal{X}_{s}$ of the nominal system. Assume that there is a local controller $h_{s}$ for the terminal state $\mathcal{X}_{f_{s}}$. The associated terminal penalty $V_{s}(\cdot)$ has the following property $\alpha_{V_{s}}\left(\left\|x_{s}\right\|\right) \leqslant V_{s}\left(x_{s}\right) \leqslant$ $\beta_{V_{s}}\left(\left\|x_{s}\right\|\right)$ for all $x_{s} \in \mathcal{X}_{f_{s}}$, where $\alpha_{V_{s}}$ and $\beta_{V_{s}}$ are class $\mathcal{K}_{\infty}$-functions. We also assume that, $\left.V_{s}\left(f_{s}\left(x_{s}, h_{s}\right)\right)\right)-$ $V_{s}\left(x_{s}\right) \leqslant-L_{s}\left(x_{s}, h_{s}\left(x_{s}\right)\right) \quad \forall x_{s} \in \mathcal{X}_{f_{s}}$ and that $V_{s}$ is Lipschitz in $\mathcal{X}_{f_{s}}$, with Lipschitz constant $L_{V_{s}}$.

\section{TRIGGERING CONDITION FOR THE DECENTRALIZED NMPC CASE}

The triggering condition for each of the subsystem of the form (26) under a decentralized NMPC control law, can be reached following a similar procedure as in the previously presented, centralized case. In the following, a similar notation as in the previous sections will be used.

Since there are mismatches between the real subsystem (26) and the nominal subsystem (30), the predicted evolution using the nominal model might differ from the real evolution of each of the subsystem. Next, a bound of the difference between the real and the predicted evolution is given. This bound depends on the error $e_{s}(k+j \mid k)$ and on the interaction and disturbance term that affects each subsystem. The error $e_{s}(k+j \mid k)$ is

$$
e_{s}(k+j \mid k)=\left\|x_{s, k+j}-\hat{x}_{s}(k+j \mid k)\right\|
$$

where $x_{s, k+j}$ is the state of the subsystem $s$, measured at time step $k+j$, and $\hat{x}_{s}(k+j \mid k)$ is the predicted state of the same subsystem at the same time step, measured from time step $k$.

Lemma 1. Consider subsystems (26) and (30), subject to the constraints (28) and assume that the previously presented Assumption 2, holds. Then, for a given sequence of inputs, the error $e_{s}(k+j \mid k)$, at time step $k+j$, will satisfy

$$
\begin{aligned}
& e_{s}(k+j \mid k) \leq L_{f_{s}}^{j-1} \cdot e_{s}(k+1 \mid k)+ \\
& +\frac{L_{f_{s}}^{j-1}-1}{L_{f_{s}}-1} \cdot\left(\sum_{i=1}^{j-1} \psi_{s}(k+i)+\sum_{i=1}^{j-1} \sum_{t=1}^{M} L_{g_{s}, t}\left\|x_{t, k+i}\right\|\right)
\end{aligned}
$$

Proof. We have

so that

$$
e_{s}(k \mid k)=\left\|x_{s, k}-\hat{x}_{s}(k \mid k)\right\|=0
$$

$$
e_{s}(k+1 \mid k)=\left\|x_{s, k+1}-\hat{x}_{s}(k+1 \mid k)\right\|
$$

and

$$
\begin{gathered}
e_{s}(k+2 \mid k)=\left\|x_{s, k+2}-\hat{x}_{s}(k+2 \mid k)\right\| \leq \\
L_{f_{s}}\left\|x_{s, k+1}-\hat{x}_{s}(k+1 \mid k)\right\|+\left\|\psi_{s}(k+1)\right\|+\left\|g_{s}\left(x_{k+1}\right)\right\| \leq \\
\leq L_{f_{s}} \cdot e_{s}(k+1 \mid k)+\left\|\psi_{s}(k+1)\right\|+\sum_{t=1}^{M} L_{g_{s}, t}\left\|x_{t, k+1}\right\| \\
e_{s}(k+3 \mid k)=\left\|x_{s, k+3}-\hat{x}_{s}(k+3 \mid k)\right\| \leq \\
L_{f_{s}}\left\|x_{s, k+2}-\hat{x}_{s}(k+2 \mid k)\right\|+\left\|\psi_{s}(k+2)\right\|+\sum_{t=1}^{M} L_{g_{s}, t}\left\|x_{t, k+2}\right\| \leq \\
L_{f_{s}} \cdot\left(L_{f_{s}} \cdot e_{s}(k+1 \mid k)+\left\|\psi_{s}(k+1)\right\|+\sum_{t=1}^{M} L_{g_{s}, t}\left\|x_{t, k+1}\right\|\right)+ \\
+\left\|\psi_{s}(k+2)\right\|+\sum_{t=1}^{M} L_{g_{s}, t}\left\|x_{t, k+2}\right\|
\end{gathered}
$$

Using similar calculations, we can derive

$$
\begin{gathered}
e_{s}(k+j \mid k) \leq L_{f_{s}}^{j-1} \cdot e_{s}(k+1 \mid k)+ \\
+\frac{L_{f_{s}}^{j-1}-1}{L_{f_{s}}-1} \cdot\left(\sum_{i=1}^{j-1} \psi_{s}(k+i)+\sum_{i=1}^{j-1} \sum_{t=1}^{M} L_{g_{s}, t}\left\|x_{t, k+i}\right\|\right)
\end{gathered}
$$

Finding the triggering condition for each of the subsystems (26), under a decentralized NMPC control law of the form (31a)-(31d), can be treated as an extension of the centralized case.

Following the procedure of the centralized case, previously presented, and with the help of Raimondo et al. [2007], it can be shown that in the same manner as in the centralized case, the triggering rule for each of the subsystems $s$, can be stated as 


$$
L_{Z_{s, j}} \cdot e_{s}(k+j \mid k-1) \leq \sigma \cdot \sum_{i=0}^{j} \alpha_{s}\left(\left\|x_{s, k-i+j}\right\|\right)
$$

and

$$
\begin{aligned}
& L_{Z_{s, j}} \cdot e_{s}(k+j \mid k-1)-\sigma \cdot \sum_{i=0}^{j} \alpha_{s}\left(\left\|x_{s, k-i+j}\right\|\right) \leq \\
& L_{Z_{s, j-1}} \cdot e_{s}(k+j-1 \mid k-1)-\sigma \cdot \sum_{i=0}^{j-1} \alpha_{s}\left(\left\|x_{s, k-i+j}\right\|\right)
\end{aligned}
$$

with $L_{Z_{s, j}}=L_{V_{s}} \cdot L_{f_{s}}^{\left(N_{s}-1\right)-j}+L_{c_{s}} \cdot \frac{L_{f_{s}}^{\left(N_{s}-1\right)-j}-1}{L_{f_{s}}-1}$.

The next OCP is triggered whenever condition (34a) or $(34 \mathrm{~b})$ is violated. Note, that the state vector $x_{k}$ is assumed to be available through measurements and that it provides the current plant information.

Theorem 3. Consider the system (26), subject to (28) under a decentralized NMPC strategy and assume that the previously presented Assumption 2 and Assumption 3 , holds. Then the NMPC control law given by (31a)(31d) along with the triggering rule (34a)-(34b), drives the closed loop system to a compact set where it is ultimately bounded.

\section{CONCLUSION}

We provided an event-triggered formulation of model predictive control based systems. The main idea is to trigger the solution of the optimal control problem only when it is needed, and not at every time-step as in the case of classic discrete time MPC. The event-based strategy is possible to alleviate the computational burden of a MPC framework. The centralized case is treated first, where we reached to an explicit event-triggered condition. Then the results has been extended to the decentralized formulation, where an event-triggered condition is given for each of the subsystems.

Future work involves finding the triggering condition in a cooperative control problem of a system of distributed agents which operate in a common environment.

\section{REFERENCES}

D.V. Dimarogonas, K.H. Johansson. Event-triggered control for multi-agent systems. 48th IEEE Conf. Decision and Control, pages $7131-7136,2009$.

W.B. Dunbar, R.M. Murray. Distributed receding horizon control with application to multi-vehicle formation stabilization. Automatica, volume 42, pages 549-558, 2006.

A. Eqtami, D.V. Dimarogonas, K.J. Kyriakopoulos. Event- triggered control for discrete-time systems. American Control Conference, pages 4719 - 4724, 2010.

E. Franco, L. Magni,T. Parisini, M. Polycarpou, D. Raimondo. Cooperative constraint control of distributed agents with nonlinear dynamics and delayed information excange: a stabilizing receding-horizon approach. IEEE Trans. Autom. Control, volume 53, pages 1:324-338, 2008.

L. Grune, F. Muller. An algorithm for event-based optimal feedback control. 48th IEEE Conf. Decision and Control, pages 5311 - 5316, 2009.
W.P.M.H. Heemels, J.H. Sandee, P.P.J. Van Den Bosch. Analysis of event-driven controllers for linear systems. International Journal of Control, volume 81, pages 4: 571-590, 2007.

Y. Iino, T. Hatanaka, M. Fujita. Event-predictive control for energy saving of wireless networked control system. American Control Conference, pages 2236-2242, 2009.

T. Keviczky, F. Borrelli, G.J. Balas. Decentralized receding horizon control for large scale dynamically decoupled systems. Automatica, volume 42, pages 13:2105-2115, 2006.

M. Lazar, W.P.M.H. Heemels. Predictive control of hybrid systems: stability results for suboptimal solutions. Automatica, volume 42, pages 1:180-185, 2009.

D. Limon Marruedo, T. Alamo, E.F. Camacho. Input-toState stable MPC for constrained discrete-time nonlinear systems with bounded additive uncertainties. 41st IEEE Conf. Decision and Control, pages 4619 - 4624, 2002.

M. Mazo Jr., and P. Tabuada. Decentralized eventtriggered control over wireless sensor/actuator networks. Submitted, 2010.

G.Pin, L. Magni, T. Parisini, D.M. Raimondo. Robust receding-horizon control of nonlinear systems with state dependent uncertainties: an input-to-state approach. American Control Conference, pages 1667 - 1672, 2008.

D.M. Raimondo, P. Hokayem, J. Lygeros, M. Morari. An iterative decentralized MPC algorithm for largescale nonlinear systems. Proceedings of the 1st IFAC Workshop on Estimation and Control of Networked Systems, pages 162-167, 2009.

D.M. Raimondo, L. Magni, R. Scattolini. Decentralized MPC of nonlinear systems: an input-to-state stability approach. International Journal of Robust and Nonlinear Control, volume 17, pages 17:1651 - 1667, 2007.

R. Scattolini. Architectures for distributed and hierarchical model predictive control - a review. Journal of Process Control, volume 19, pages 723-731, 2009.

J. Sijs, M. Lazar, W.P.M.H. Heemels. On integration of event-based estimation and robust MPC in a feedback loop. HSCC '10:Proc. of the 13th ACM International Conference on Hybrid Systems: Computation and Control, pages 31-40, 2010.

P. Tabuada. Event-triggered real-time scheduling of stabilizing control tasks. IEEE Transactions on Automatic Control, volume 52, pages 9:1680-1685, 2007.

P. Varutti, R. Findeisen. On the synchronization problem for the stabilization of networked control systems over nondeterministic networks. American Control Conference, pages 2216 - 2221, 2009.

X. Wang, M.D. Lemmon. Event-triggering in distributed networked systems with data dropouts and delays. HSCC '09:Proc. of the 12th International Conference on Hybrid Systems: Computation and Control, volume 52, pages 366-380, 2009. 